\title{
Theoretical and Experimental Investigations for the Virtual Mass of a Taylor Bubble
}

\author{
Abdullah Abbas Kendoush', Warren E. Overton² \\ ${ }^{1}$ Dept. of Nuclear Engineering Technology \\ akendoush@augustatech.edu \\ ${ }^{2}$ Dept. of Mechanical Engineering Technology \\ warren.overton@augustatech.edu \\ Augusta Technical College, Augusta, GA 30906, USA
}

\begin{abstract}
An exact theoretical analysis was presented for the virtual mass of the Taylor bubble. The present theoretical results were validated experimentally and proved the earlier results of Kendoush [1] were grossly approximate. An experiment was designed, installed, and tested for the purpose of obtaining the virtual mass by using a 3-D manufactured polymeric Taylor bubble.
\end{abstract}

Keywords: Slug flow, Taylor bubble, Virtual mass coefficient, Multiphase Flow, Capillary number, Transient Bubbly Flow

\section{Introduction}

Unsteady motion of bubbles arise in many engineering applications, ranging from boiling to adiabatic two-phase flow in pipes. Slug flow that contains Taylor bubbles is considered as one of the two-phase flow regimes flowing in pipelines.

When the velocity of Taylor bubble is changing under accelerating flow, a force called the virtual mass (or added mass) force is generated [2]. This force arises from the acceleration of the mass of the liquid that surrounds the Taylor bubble. Bubble shape affects the value of the virtual mass coefficient $C_{m}$, for example, a spherical bubble has $C_{m}=0.5$ [3]. A formulated form of $C_{m}$ was obtained for the spherical-cap bubble [4 \& 5] and the oblate-ellipsoidal bubble [6].

It is always advisable to verify the theoretical results. Kendoush et al. [7] did so for the $C_{m}$ of two solid spheres accelerating in fluids and found good agreement with the theories of other investigators. An excellent review on the subject was presented by Michaelides [8]. Hanau and Raithby [9] derived equations for the virtual mass and drag coefficients of a Taylor bubble moving in a horizontal pipe and found that the $C_{m}$ was a function of bubble geometry.

Takhistov et al. [10] performed experiments on the flow of Taylor air bubbles in $\mathrm{KCl} / \mathrm{H}_{2} \mathrm{SO}_{4}$ solutions in a capillary tube of $0.25 \mathrm{~mm}$ radius under the influence of an electric field. They measured the time dependent bubble velocity and found it to be a function of bubble length in contrast to the steady state velocity which is a function of pipe radius. bubble.

The aim of the present work is to verify experimentally, the theoretical results of the $C_{m}$ obtained here for the Taylor

\section{Theoretical Analysis}

\subsection{The virtual mass force on the Taylor bubble}

2.1.1 The bubble nose region

The velocity potential of a Taylor bubble rising with velocity $U$ in a circular pipe of radius $R$ is given by Clanet et al. [11] in cylindrical (y,r) coordinates (Fig. 1) as follows

$$
\varnothing=\mathrm{yU}+\mathrm{A}_{\mathrm{o}} \mathrm{e}^{\mathrm{ky}} \mathrm{J}_{0}(\mathrm{kr})
$$

Where $\mathrm{J}_{0}$ is the Bessel function of order zero. The first term of this equation represents the 


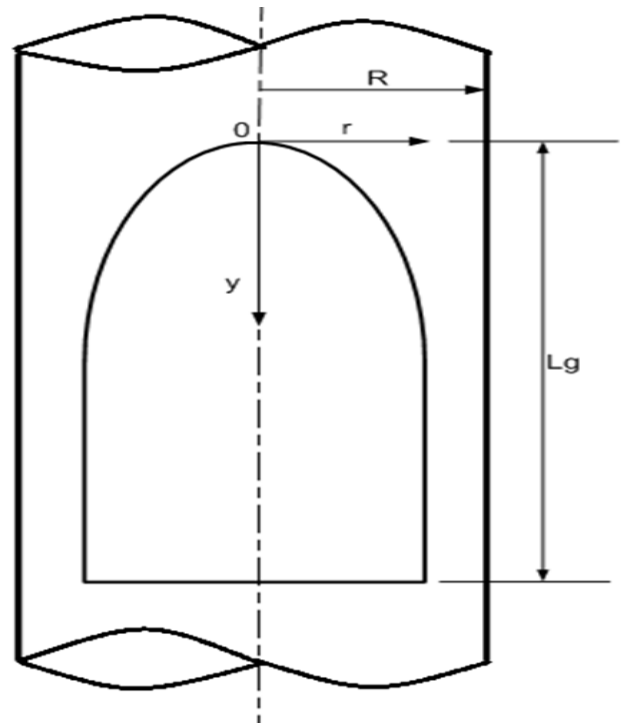

Fig. 1: Schematic diagram of the Taylor bubble.

uniform flow. Clanet et al. [11] assumed that the velocity far ahead of the bubble is equal to the bubble velocity. The two constants $\mathrm{A}_{\mathrm{o}}$ and $\mathrm{k}$ are given as follows

$$
A_{o}=-\frac{U}{k} \quad \text { and } \quad k=\frac{3.83}{R}
$$

The associated stream function is

$$
\psi=\frac{r U}{k}\left(e^{k y} J_{1}(k r)-\frac{1}{2} k r\right)
$$

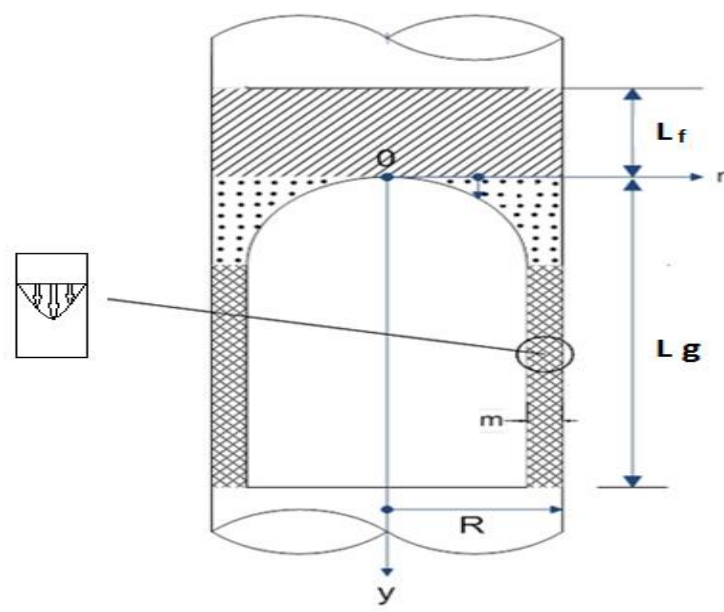

Fig. 2: Partitions of the various flow regions around the Taylor bubble. 
where $J_{1}$ is the Bessel function of order one. The domain of the forward region of the Taylor bubble is bounded as $-\mathrm{L}_{\mathrm{f}}<\mathrm{y}<\mathrm{L}_{\mathrm{g}}$, where $\mathrm{L}_{\mathrm{f}}$ is the length of the liquid region and $\mathrm{L}_{\mathrm{g}}$ is the length of the slug bubble as shown in Fig. 2.

Removing the uniform flow vector $y U$ from Eq. (1) makes the Taylor bubble rise with velocity $U$ in a mass of still liquid, hence we get

$$
\emptyset=-\left(\frac{U}{k}\right) e^{k y} J_{0}(k r)
$$

The velocity components of the bubble are given as follows

$$
V_{r}=-\frac{\partial \emptyset}{\partial r} \quad \text { and } \quad V_{y}=-\frac{\partial \emptyset}{\partial y}
$$

Since $\frac{d J_{0}(r)}{d r}=-J_{1}(r)$, the above equations become

$$
V_{r}=-U e^{k y} J_{1}(k r)
$$

and

$$
V_{y}=-U e^{k y} J_{0}(k r)
$$

The forward region of the bubble may be divided into the following two subregions, namely; a region near the bubble nose (shown dotted in Fig. 2), and the thin layer liquid region between the pipe wall and bubble cylindrical surface. These features appear to be common to the physical phenomena that are classed as a slug flow regime.

The length of the Taylor bubble is divided into two parts, a small part containing the rounded bubble nose and the remaining large cylindrical length of the bubble (bounded by cross-hatching in Fig. 2). Here $\mathrm{m}=1.34 \mathrm{RCa}^{2 / 3}$, is the thickness of the thin liquid layer between the pipe wall and the cylindrical surface of the bubble as derived by Bretherton [12]. Here Ca represents the capillary number $(\mathrm{Ca}=\mu \mathrm{U} / \sigma)$, where $\mu$ is the dynamic viscosity of the liquid and $\sigma$ is the surface tension. The capillary number gives the ratio of the viscous force to the force of surface tension. The kinetic energy of the liquid in this region is obtained as follows

$$
T_{a}=\frac{1}{2} \rho \int_{r=0}^{r=R} \int_{h y=0}^{y=H(r)}\left(V_{r}^{2}+V_{y}^{2}\right) d \forall
$$

where $\mathrm{d} \forall=2 \pi \mathrm{rdrdy}$.

Here $\rho$ is the density of the liquid. The function H(r) in this equation is obtained as follows; the equation of the surface of the bubble can be obtained from the stream function Eq. (3) namely,

$$
\frac{r U}{k}\left(e^{k y} J_{1}(k r)-\frac{1}{2} k r\right)=0
$$

The solution of this equation for $\mathrm{y}$ gives $\mathrm{y}=\mathrm{H}(\mathrm{r})$, as follows

$$
H(r)=\frac{1}{k}\left[\ln \frac{1}{2} k r-\ln J_{1}(k r)\right]
$$


The elemental volume of the bubble is given as follows $d \forall=2 \pi r d r d y$. Substituting Eqs. (6) and (7) into Eq. (8) and carrying out the integration, gives

$$
T_{a}=\frac{\pi \rho U^{2}}{2 k}\left(\int_{0}^{R} 0.25 k^{2} r^{3} d r-\int_{0}^{R} r J_{1}^{2}(k r) d r+\int_{0}^{R} \frac{0.25 k^{2} r^{3} J_{0}^{2}(k r)}{J_{1}^{2}(k r)} d r-\int_{0}^{R} r J_{0}^{2}(k r) d r\right)
$$

Note that

$$
\int r J_{0}^{2}(k r) d r=\frac{1}{2} r^{2}\left[J_{0}^{2}(k r)+J_{1}^{2}(k r)\right]+C 1
$$

and

$$
\int r J_{1}^{2}(k r) d r=\frac{1}{2} r^{2}\left[J_{1}^{2}(k r)-J_{0}(k r) J_{2}(k r)\right]+C 2
$$

where C1 and C2 are constants. There is no analytical solution for the third integral from the left side of Eq. (11), therefore, it was numerically integrated after introducing the non-dimensional parameter $\eta=\mathrm{r} / \mathrm{R}$ as follows

$$
\frac{(3.83 R)^{2}}{4} \int_{0}^{1} \eta^{3} \frac{J_{0}^{2}(3.83 \eta)}{J_{1}^{2}(3.83 \eta)} d \eta=554.724 R^{2}
$$

Substituting this equation into Eq. (11) and integrating, we get after lengthy algebra

$$
T_{a}=\frac{\pi \rho U^{2}}{2 k}\left(0.25 k^{2} \frac{R^{2}}{4}-R^{2} J_{1}^{2}(k R)+\frac{R^{2}}{2} J_{0}(k R) J_{2}(k R)+554.724 R^{2}-\frac{R^{2}}{2} J_{0}^{2}(k R)\right)
$$

Substituting the values of Bessel functions) and rearranging, we get

$$
T_{a}=72.496 \pi \rho U^{2} R^{3}
$$

\subsubsection{The thin liquid layer region}

The thin liquid layer region is shown cross hatched in Fig. 2. The kinetic energy of the liquid in this region is very small in comparison with the bubble nose region due to the smaller mass of the liquid there. This means that the contribution from this region to the virtual mass coefficient is minimum. Bubble mass flow up equals displaced water mass flow down. Measured bubble velocity in $40 \mathrm{~mm}$ tube averaged $0.212 \mathrm{~m} / \mathrm{s}$ up. Bubble bottom cross sectional area is 18.86 times the thin film cross-sectional area. Thin film velocity: $18.86(0.212 \mathrm{~m} / \mathrm{s})=4.0 \mathrm{~m} / \mathrm{s}$ down.

\subsubsection{The wake region of the Taylor bubble}

The liquid flow in the wake region of the Taylor bubble may be assumed inviscid, vortical and confined to the spherical Hill vortex as shown in Fig 3. Parlange [13], Kendoush [14] and Milne-Thomson [3] utilized 


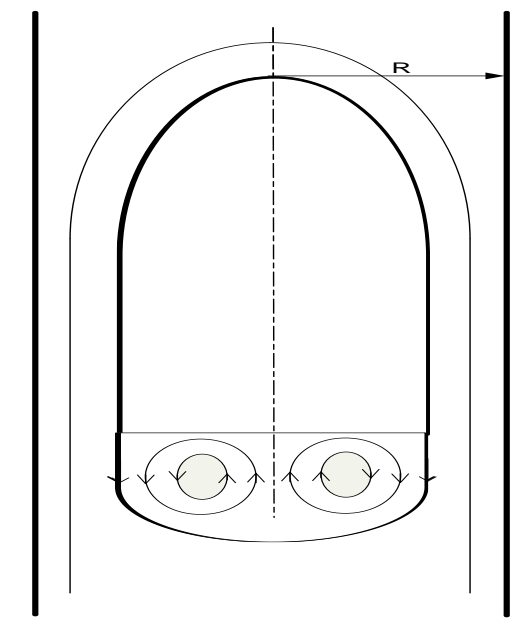

Fig. 3: A spherical Hill vortex at the wake of the Taylor bubble.

a similar model to obtain information about parameters of the wake of a spherical - cap bubble. Nakoryakov et al. [15] observed toroidal vortex in the wake of the Taylor bubble. Apply the polar $(r, \theta)$ coordinates in the wake region $L_{f}$ of Fig. 2. The following velocity field was used

$$
V_{r w}=\frac{3}{2} U_{w}\left(\frac{r}{R}\right)^{2} \cos \theta
$$

and

$$
V_{\theta w}=3 U_{w}\left(\frac{r}{R}\right)^{2} \sin \theta
$$

where $U_{w}$ is the main liquid velocity in the wake.

The kinetic energy of the liquid of the wake is given as follows

$$
T_{b}=\frac{1}{2} \rho \int_{0}^{R} \int_{\pi / 2}^{\pi}\left(V_{r w}^{2}+V_{\theta w}^{2}\right) 2 \pi r^{2} \sin \theta d r d \theta
$$

Substituting Eqs. (17) and (18) into this equation gives

$$
T_{b}=9 \pi \rho U_{w}^{2} \int_{0}^{R} \int_{\pi / 2}^{\pi}\left(\frac{1}{4}\left(\frac{r}{R}\right)^{4} \cos \theta^{2}+\left(\frac{r}{R}\right)^{4} \sin \theta^{2}\right) r^{2} \sin \theta d r d \theta
$$

The evaluation of this integral gives

$$
T_{b}=\frac{27}{28} \pi \rho U_{w}^{2} R^{3}
$$

The relationship between the liquid wake velocity and the main liquid or bubble velocity is recommended by Garimella et al. [16] as follows 


$$
U=1.2 U_{w}
$$

When this equation is substituted in Eq. (21), we got the following

$$
T_{b}=0.6696 \pi \rho U^{2} R^{3}
$$

The wake region is not a fully liquid region, some small spherical bubbles may exist there, but we shall assume it a fully liquid region. The total kinetic energy imparted to the liquid due to the motion of the Taylor bubble would be the sum of the kinetic energy of the liquid at the forward region plus that at the wake region of the bubble, which is

$$
T=T_{a}+T_{b}
$$

Substituting Eqs. (16) and (23) into this Equation, gives

$$
T=72.496 \pi \rho U^{2} R^{3}+0.6696 \pi \rho U^{2} R^{3}
$$

This kinetic energy may also be given by the following

$$
T=\frac{1}{2} M U^{2}
$$

where $\mathrm{M}$ is the mass of liquid displaced by the bubble. We get the following from Eqs. (25) and (26)

$$
M=146.3292 \pi \rho R^{3}
$$

The virtual mass coefficient is defined by the following

$$
C_{m}=\frac{\text { The volume of the "virtual mass" }}{\text { volume displaced by bubble }}
$$

The volume displaced by the bubble is defined for the Taylor bubble of Fig. 1 as follows (a detailed derivation is given in Appendix A)

$$
\forall=\pi R^{3} f^{2}(\mathrm{Ca})\left[\frac{L_{g}}{R}-\frac{1}{3} f(\mathrm{Ca})\right]
$$

Here $\mathrm{f}(\mathrm{Ca})$ is given by Appendix A. Accordingly, $\mathrm{C}_{\mathrm{m}}$ becomes equal to the following

$$
C_{m}=\frac{146.3292}{(f(C a))^{2}\left[\frac{L g}{R}-\frac{1}{3} f(C a)\right]}
$$

This equation indicates that the virtual mass coefficient is a function of the geometry of the Taylor bubble, and the capillary number of the flow. Figure 4 shows the variation of the virtual mass coefficient with the capillary number at constant values of $\frac{\mathrm{Lg}_{\mathrm{g}}}{\mathrm{R}}$. No significant change in the function is seen for $\mathrm{Ca}<0.001$. Figure 5 shows the variation of the virtual mass coefficient with the geometrical ratio of the Taylor bubble at constant values of the capillary number. 


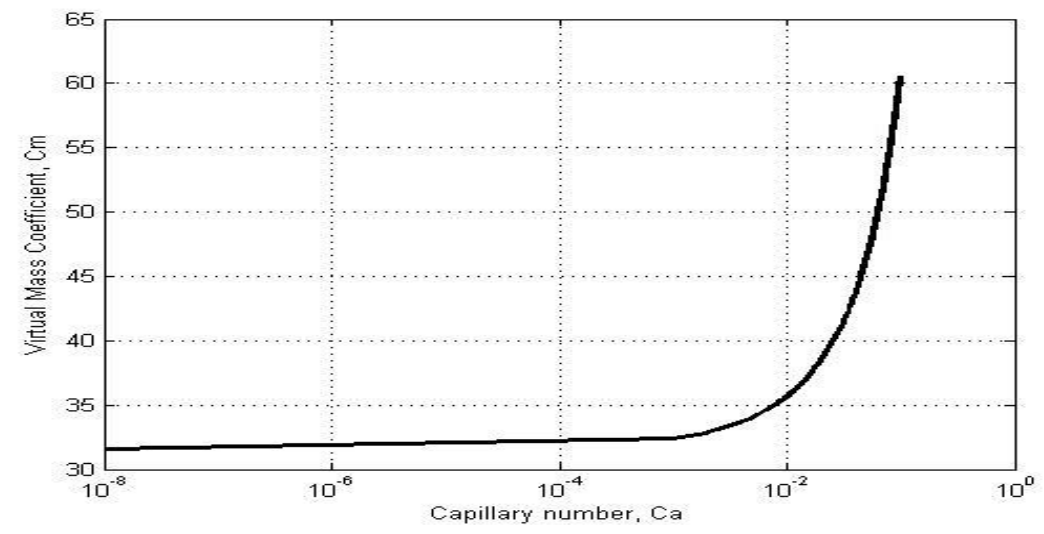

Fig. 4: The variation of the virtual mass coefficient of a Taylor bubble with the capillary number at $\mathrm{L}_{\mathrm{g}} / \mathrm{R}=5$.

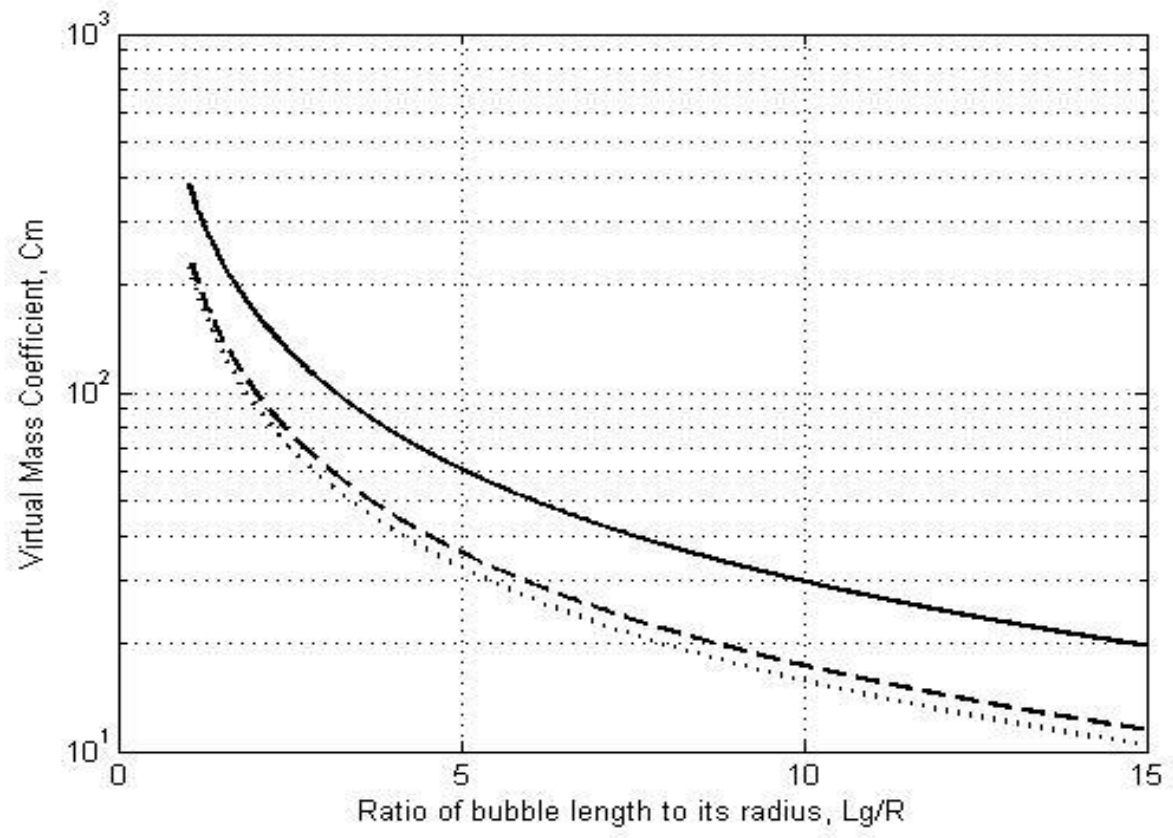

Fig. 5: The variation of the virtual mass coefficient with the geometrical ratio of the Taylor bubble and the capillary number, $\left(\ldots\right.$ ) $\mathrm{Ca}=0.1,{ }_{\ldots} \ldots$ ) $\mathrm{Ca}=0.01,(\ldots) \mathrm{a}=0.001$.

The distribution of the various flow regimes around Taylor bubble has important effects on the virtual mass coefficient. The higher contribution (99.1\%) comes, from the liquid motion at the bubble nose, and the lower contribution $(0.9 \%)$ comes from the effect of the wake region. This is mainly due to the low vortical velocity of the liquid there (see Eq. (22)).

The dependence of $\mathrm{C}_{\mathrm{m}}$ in Eq. (30) on bubble geometry is in agreement with Hanau and Raithby [9] mentioned earlier. 


\section{Experimental Simulation of the Taylor Bubble}

The experimental apparatus shown in Fig.6 simulates the Taylor bubble by using an ABS polymer model. The model was designed and fabricated on a 3D printer (StrataSys Dimension SST 1200). We have chosen two different sizes matching the Taylor bubble based on our earlier experiments on air Taylor bubble (aTb) [18].

The experiments were conducted in a transparent Plexiglas column of $1.219 \mathrm{~m}$ height and a square cross-section of $15 \mathrm{~cm}$ to reduce the distortion due to optical refraction while capturing high-speed photographs of the travel of the plastic Taylor bubble (pTb) inside the cylindrical pipe centered in the square cross-sectional containment column.

The fishing line connected to the pTb was run across two pulleys to a reel driven by the programmable VFD motor (Fig. 6). Pulley 1 is supported by a Newton scale to measure the force required to pull the pTb to the accuracy of $\pm 0.25 \mathrm{~N}$. Note: The required force equals $1 / 2$ the value shown on the Newton scale.

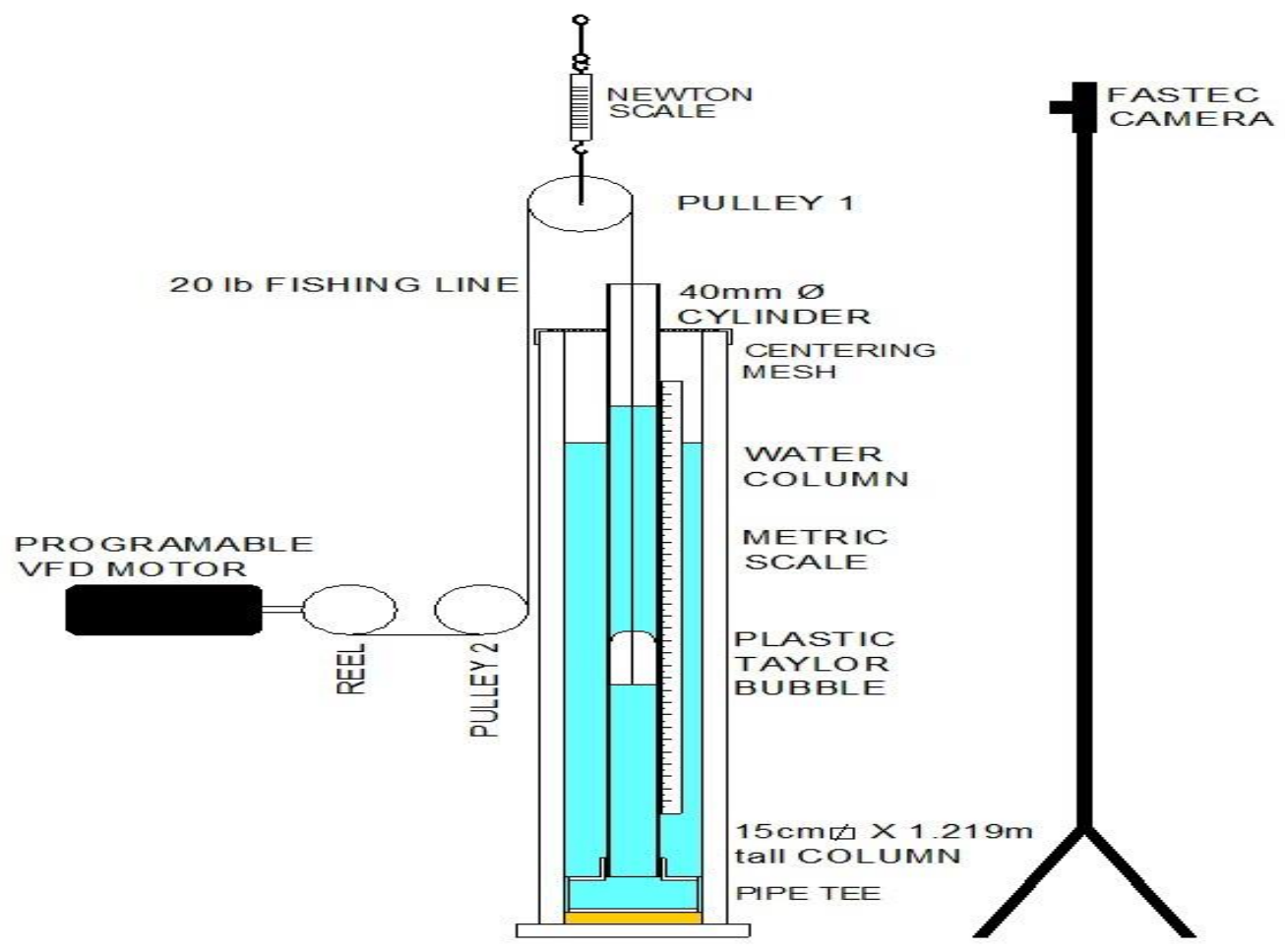

Fig. 6: Schematic diagram of the experimental apparatus. VFD stands for Variable Frequency Drive.

Our previous experimental results [18] and the results of Liao and Zhao [19] showed a gradual decrease of the liquid film thickness, so the shape of the Taylor bubble possesses sharpness of the bubble nose. The work of Lertnuwat [20] also appreciated this shape.

Great care was taken to smooth the surface of the $\mathrm{pTb}$ in order to reduce the friction as much as possible. It should be noted that the pTb floats in water and this requires the addition of extra weights of metallic washers adhered to the base of the $\mathrm{pTb}$ to make it neutrally buoyant in water.

A high-speed camera (Fastec Imaging) at 250 frames per second and 9 mega-pixel resolution, was employed to measure the force recorded on the Newton scale. This force reflects two times the tensile force required to lift the pTb. 


\section{Validation and Discussion}

The general equation of motion is given as follows [22]

$$
V_{s}\left(\rho_{s}+C_{m} \rho_{f}\right) \frac{d U}{d t}=F-V_{s}\left(\rho_{s}-\rho_{f}\right) g-\frac{1}{2} C_{D} \pi \rho_{f} R^{2} U^{2}
$$

The history term was neglected in this equation as its contribution is extremely small [22]. Here F represents the force registered by the Newton scale (Fig. 6). This force is normally halved due to the use of the pulley. The second term on the right side of Eq. (31) represents buoyancy and the third term represents the drag force.

The drag coefficient $C_{D}$ of the Taylor bubble was obtained from [24] as follows

$$
C_{D}=8.1657 \frac{L_{g}}{R}-2.722 \quad \text { for } C_{a}<0.005
$$

This equation was compared with the experimental data of Kawaji et al. [25] and Tudose [26] who also used solid plastic models of the Taylor bubble and the ratio of their experimental drag to that given by the above equation was 1.8 (Table 1). This ratio was used in Eq. (31) to get $\mathrm{C}_{\mathrm{m}}=40.4$ for the $40 \mathrm{~mm}$ diameter pTb model as shown in Fig. 7 where the agreement between theory and experiment is remarkable.

The present results showed how the experiment became the fair judge for the validity of the theory. The earlier theoretical results [1] has the approximation of expanding the velocity components of the fluid around the apex of the bubble $(\mathrm{y}=0)$ in Eqs. (6 and 7). This produced a grossly approximate value of $\mathrm{C}_{\mathrm{m}}$ that did not agree with the present experimental results. Both the current and the previous [1] solutions share the dependence of $\mathrm{C}_{\mathrm{m}}$ on the Taylor bubble geometry $\left(\frac{\mathrm{Lg}}{\mathrm{R}}\right)$.

Table 1: Comparison with the experimental data of Tudose [26].

\begin{tabular}{|c|c|c|c|c|}
\hline$L_{g}, \mathrm{~cm}$ & $\frac{L_{g}}{R}$ & $\begin{array}{c}\text { Calculated } C_{D} \\
\text { Eq. (32) }\end{array}$ & Experimental $C_{D}$ & Calculated $C_{m}$ \\
\hline 7.5 & 5.86 & 36.33 & 66.7 (Fig. 5.2.5) & 28.033 (Eq. 30) \\
\hline 15 & 11.72 & 77.8 & 137 (Fig. A.1.4) & 13.536 (Eq. 30) \\
\hline 9.5 & 4.750 & 36.07 & 36.68 & 32.452 (Eq. 31) \\
\hline 6.1 & 2.461 & 16.81 & 16.69 & 191.182 (Eq. 31) \\
\hline
\end{tabular}




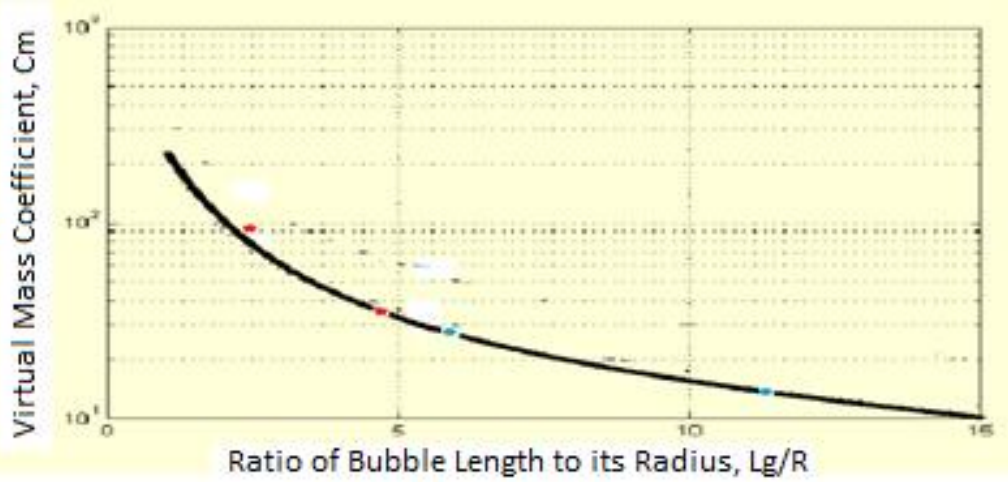

Fig. 7: Comparison between the current theory (Eq. (30)) solid line with $C_{a}=0.003$, Eq. (30) blue circular points, and Eq. (31) red star points.

\section{Conclusions}

New theoretical analyses were performed and produced a solution for the virtual mass coefficient of the Taylor bubble. The new solution proved to be superior to that obtained earlier [1] when comparing both solutions to the present experimental results.

Modern 3D printer was used to manufacture a solid shape similar to the Taylor bubble. An experimental system was designed, installed, and tested to accelerate the solid model of Taylor bubble to register its velocity and force by using a high-speed camera and a precise Newton scale.

\section{Acknowledgements}

Thanks to Mr. Charlie Nesbitt of Southern Nuclear Company for the support. Thanks also to Ms. Kim Gaines, Dr. David M. Maryniak, and Ms. Surekha Prasad and the student experimenters Jacob L. Brainard, Hunter Garrett, and Joshua R. Robertson for their efforts and support.

\section{References}

[1] A. A. Kendoush, "The Virtual Mass Theory of a Taylor Bubble Rising in Vertical Pipes," ASME J. Fluids Eng., vol. 140, pp. 051202-1-051202-6, 2018.

[2] G.K. Batchelor, An Introduction to Fluid Dynamics, Cambridge University Press, Cambridge, UK, 1967.

[3] L. M. Milne-Thomson, Theoretical Hydrodynamics, 5th Ed., Macmillan, London, 1960.

[4] A. A. Kendoush, "The Virtual Mass of a Spherical-Cap Bubble," Phys. Fluids, vol. 15, no. 9, pp.2782-2785, 2003.

[5] A. A. Kendoush, "Erratum: The Virtual Mass of a Spherical-Cap Bubble," Phys. Fluids, vol.16, no.7, p.2713, 2004.

[6] A. A. Kendoush, "The Virtual Mass of an Oblate-Ellipsoidal Bubble," Phys. Lett. A, vol. 366, no. 3, pp. 253-255, 2007.

[7] A. A. Kendoush, A. H. Sulaymon, and S. A. M. Mohammed, "Experimental Evaluation of the Virtual Mass of Two Spheres Accelerating in Fluids." Exp. Therm. Fluid Sci., vol. 31, pp. 813-823, 2007.

[8] E. E. Michaelides, "Review - The Transient Equation of Motion for Particles, Bubbles, and Droplets," ASME J. Fluids Eng., vol. 119, no. 2, pp. 233-247, 1997.

[9] V. D. Hanau and G. D. Raithby, "A Transient Two-Fluid Model for the Simulation of Slug Flow in Pipelines-I: Theory," Int. J. Multiphase Flow, vol. 21, no. 3, pp. 335-349, 1995.

[10] P. Takhistov, A. Indenikina, and H. -C Chang, "Electrokinetic Displacement of Air Bubbles in Micro Channels," Phys. Fluids, vol. 14, no. 1, pp.1-14, 2002.

[11] C. Clanet, P. Heraud, and G. Searby, "On the Motion of Bubbles in Vertical Tubes of Arbitrary Cross-Sections: Some Complements of the Dumitrescu-Taylor Problem”, J. Fluid Mech., vol. 519, pp. 359-376, 2004. 
[12] F. P. Bretherton, "The Motion of Long Bubbles in Tubes," J. Fluid Mech., vol. 10, pp.166-188, 1961.

[13] J. -Y. Parlange, "Spherical-Cap Bubbles with Laminar Wakes," J. Fluid Mech., vol. 37, pp.257-263, 1969.

[14] A. A. Kendoush, "Theory of Convective Heat and Mass Transfer to Spherical-Cap Bubbles," AIChE Journal, vol. 40, pp.1440-1448, 1994.

[15] V. E. Nakoryakov, O. N. Kashinsky, A. V. Petukhov, and R. S. Gorelik, "Study of Local Hydrodynamic Characteristics of Upward Slug Flow," Experiments in Fluids, vol. 7 , pp. 560-566, 1989.

[16] S. Garimella, J. D. Killion, and J. W. Coleman, “ An Experimentally Validated Model for Two-Phase Pressure Drop in the Intermittent Flow Regime for Circular Micro channels. ASME J. Fluids Eng., vol. 124, pp. 205-214, 2002.

[17] D. Dumitrescu, D., "Stromung an Einer Luftblase im Senkrechten Rohr," Z. Angew Math. Mech., vol. 23, pp. 139$149,1943$.

[18] A. A. Kendoush, K. W. Gaines, and W. E. Overton, "Drag Forces on a Taylor Bubble Rising Steadily in Pipes," Sent for publication.

[19] Q. Liao and T.S. Zhao, "Modelling of Taylor Bubble Rising in a Vertical Mini Noncircular Channel Filled with a stagnant liquid," Int. J. Multiphase Flow, vol. 29, pp. 411-434, 2003.

[20] B. Lertnuwat, "Shapes of an Air Taylor Bubbles in Stagnant Liquids Influenced by Different Surfaces Tensions," Int. J. Applied Mechanics \& Eng., vol. 23, no.1, pp. 79-90, 2018.

[21] S. Ishutov, "3D printing porous proxies as a new tool for laboratory and numerical analyses of sedimentary rocks," $\mathrm{Ph} . \mathrm{D}$. Thesis, Iowa State University, 2017.

[Online]. Available: https://lib.dr.iastate.edu/cgi/viewcontent.cgi?article=6545\&context=etd

[22] R. Clift, J. R. Grace, and M. E. Weber, Bubbles, Drops, and Particles, Academic Press, New York, 1978.

[23] M. Pierucci, "Velocity of Cavitation Bubbles in Uniform Flow field High and Low Reynolds Number," AIAA J., vol. 29, pp.505-514, 1991.

[24] A. A. Kendoush, "Drag Forces on a Taylor Bubble Rising Steadily in Pipes-Theory," Sent for publication.

[25] M. Kawaji, J. M. DeJesus, and E.T. Tudose, "Investigation of Flow Structures in Vertical Slug Flow," Nucl. Eng. Des. vol. 175, pp. 37-48, 1997.

[26] E. T. Tudose, "Experimental investigation of Taylor bubble acceleration mechanism in slug flow," Master Thesis, Department of Chemical Engineering and Applied Chemistry, University of Toronto, 1997. [Online]. Available: http://www.collectionscanada.gc.ca/obj/s4/f2/dsk2/ftp01/MQ28827.pdf

\section{APPENDIX A: DERIVATION OF EQ. (37)}

\section{The volume of liquid displaced by the Taylor bubble of Fig. 1}

Note that the thickness of the thin liquid layer is given by the following equation of Bretherton [12]

$$
\mathrm{m}=1.34 \mathrm{RCa}^{2 / 3} \quad \text { (A1) }
$$

The volume of liquid displaced by the Taylor bubble is given as a cylinder and a hemisphere as follows

$$
\forall=\pi(\mathrm{R}-\mathrm{m})^{2}\left[\mathrm{~L}_{\mathrm{g}}-(\mathrm{R}-\mathrm{m})\right]+\frac{2 \pi}{3}(\mathrm{R}-\mathrm{m})^{3}
$$

Here, the bubble nose was assumed spherical as was considered by Dumitrescu [17]. Substituting Eq. (A1) into Eq. (A2), gives the following after some lengthy algebra

where

$$
\forall=\pi \mathrm{R}^{3} \mathrm{f}^{2}(\mathrm{Ca})\left[\frac{\mathrm{Lg}}{\mathrm{R}}-\frac{1}{3} \mathrm{f}(\mathrm{Ca})\right]
$$

$$
\mathrm{f}(\mathrm{Ca})=1-1.34 \mathrm{Ca}^{2 / 3}
$$

